\title{
Plasma Actuator with Two Mesh Electrodes to Control the Flow Boundary Layer
}

\author{
Ernest Gnapowski' \\ 1 Department of Transport, University College of Enterprise and Administration, 20-150 Lublin, Poland \\ e-mail:egnapowski@gmail.com
}

\begin{abstract}
The manuscript presents selected designs of Dielectric Barrier Discharge (DBD) plasma actuators used to control the flow of the boundary layer on the surface of the wing. The principle of DBD plasma actuator operation and the process of the "ion wind" formation are presented. The manuscript presents the results of the tests carried out on the sash model with the SD 7003 profile and a DBD plasma actuator with two mesh electrodes, for which tests were carried out and tunnel images were recorded.
\end{abstract}

Keywords: plasma actuator, DBD discharge, high lift device, lift force.

\section{INTRODUCTION}

Numerous experimental studies carried out by the pioneers of aviation helped to gain the experience and skills necessary for the construction of aircrafts. The structural solutions related to the airfoil can be divided into two main groups: the systems that increase the lift force by using mechanical elements called wing mechanization and the non-mechanical ones that influence the air circulation in the boundary layer. The elements that increase the lift force allow for optimization and increase the flight safety, especially during take-off or landing when the wing is working at large angles of attack. These systems increase the lifting force, preventing the detachment of the air stream from the wing's profile, preventing stall and loss of the lift force. The manuscript describes the selected designs of Dielectric Barrier Discharge (DBD) plasma actuators that allow changing the air flow in the boundary layer of the wing. Tunnel investigations of a plasma actuator with two mesh electrodes are presented.

\section{BASIC PRINCIPLE OF DBD PLASMA ACTUATOR}

Currently, there are many design solutions in aviation that allow increasing the wing lift. The main element influencing the change of wing support force is the selection of the aerodynamic profile. The choice of the profile depends on the type and purpose of the aircraft. Even a properly selected wing profile loses the lift under certain conditions (especially at large angles of attack). Currently, to prevent separation of air streams from the wing profile, systems called wing mechanisms (mechanical elements) are used, which include: slats, flaps, front flaps, flaperon [1-6] and non-mechanical non-mechanical): wing fence, dogtooth, notch leading edge, LERX, wings cuffs, vortilons, vortex generator [7-10].

DBD plasma actuators constitute a new solution for controlling the boundary layer. The design of the DBD plasma actuator is quite simple, it consists of two flat electrodes (metal / copper strips) separated by a dielectric. One of the electrodes on the surface of the wing is in direct contact with 
the flowing air. The second electrode is completely covered with a dielectric. Both electrodes are connected to a high voltage power supply system. The use of the dielectric prevents spark or arc discharge which cause high local temperature rise [11-12]. The most common configuration of the DBD plasma actuator is an asymmetrical system, consisting of flat parallel electrodes separated by a dielectric layer, as shown in Figure 1.

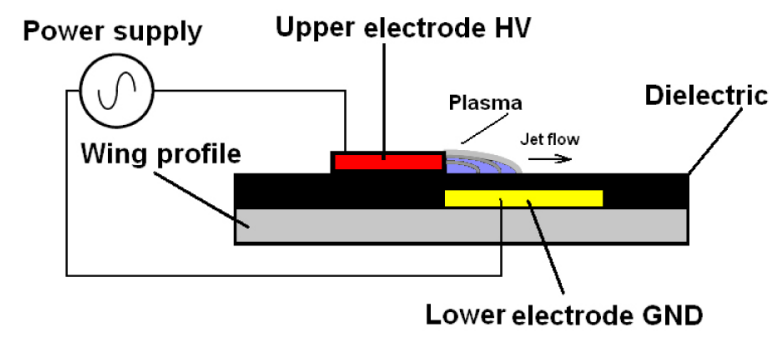

Fig. 1. Design of a DBD plasma actuator with an asymmetrical electrode system

During the operation of the DBD plasma actuator, the electrodes are supplied with high voltage (several / tens $\mathrm{kV}$ ), the effect of the system is ionized air (plasma) that moves from the exposed electrode towards the one covered with a dielectric. During the movement of the plasma (ionized air), the plasma and inert air molecules collide. As a consequence of the elastic collisions between the moving charged molecules and inert gas molecules, they increase their rate, causing an "electric wind" that occurs in close proximity to the wing surface. The air surrounding the upper electrode is ionized, attracted to the charged surface of the dielectric, thus forming a wall jet. By adjusting the direction of the wall stream resulting from the DBD plasma system, it is possible to create strong vortices, similar to those obtained by vortex generators, as shown in Figure 2. One of the main mechanisms influencing the flow exerted by the DBD plasma actuator is the generation of "ion wind" [13-14]. During the op- eration of the DBD system, ozone is generated in the air. The ozone is a byproduct of air ionization (oxygen) by plasma DBD systems; it is a strong oxidant that oxidizes the metal electrodes. Copper easily forms oxides in the air; thus, in plasma this oxidation is much stronger. The resulting oxides reduce the conductivity of the electrodes and decrease the efficiency of the DBD system. The electrodes made of stainless steel should be used for testing (they do not oxidize).

The use of DBD plasma actuators on the surface of the wing allows for increasing the angle of attack and delays stall. The DBD system, in comparison with other systems, does not have any moving parts such as tendons, valves, diaphragms, cylinders or gears, in order to activate it. This prevents the design problems affecting the construction of the aircraft. DBD plasma actuators can be used to actively control the flow of aircraft, easily adapting to their operating conditions [15]. Plasma actuators are much faster than any currently available systems. DBD systems have a very short response time, amounting to fractions of a second.

\section{SELECTED STRUCTURES OF BDB PLASMA ACTUATORS}

The research on the use of plasma actuators for flow control is carried out by many research centers around the world. Numerous modifications are being introduced, among others; changes in the shape of electrodes [16], modification of the power supply system (frequency, voltage, power), number and placement of electrodes on the wing. Most of the experimental tests carried out are with an asymmetrical configuration of electrodes. Such configurations are presented in Figure 3.

Experimental studies using multi-electron systems in an asymmetrical system [18] were also carried out, as shown in Figure 4. (a)

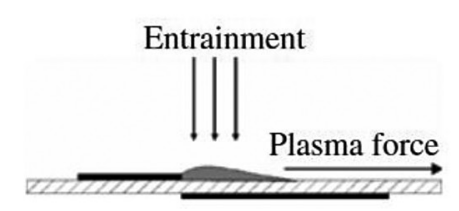

(b)

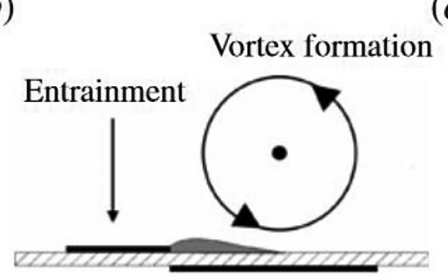

(c)

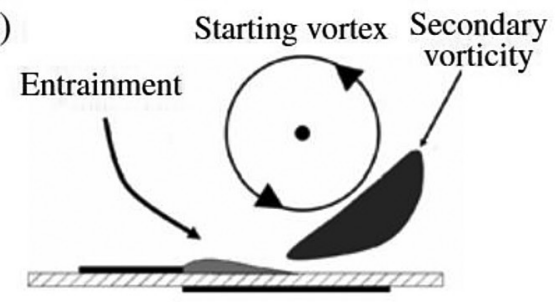

Fig. 2. The development of the starting point vortex (a) plasma initiation (b) vortex formation,

(c) generation of secondary vorticity [13] 


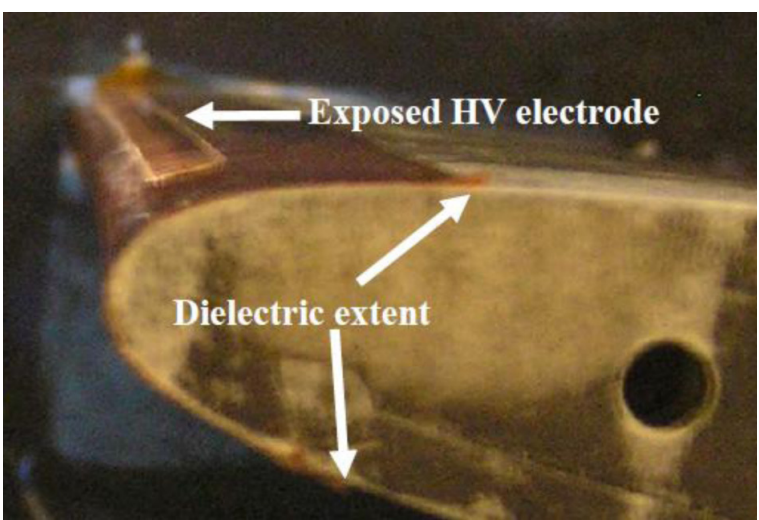

Fig. 3. Photograph of the DBD plasma actuator mounted near the airfoil leading edge in reverse upstream arrangement located $6 \mathrm{~mm}$ in arc length from $x / c=0[17]$

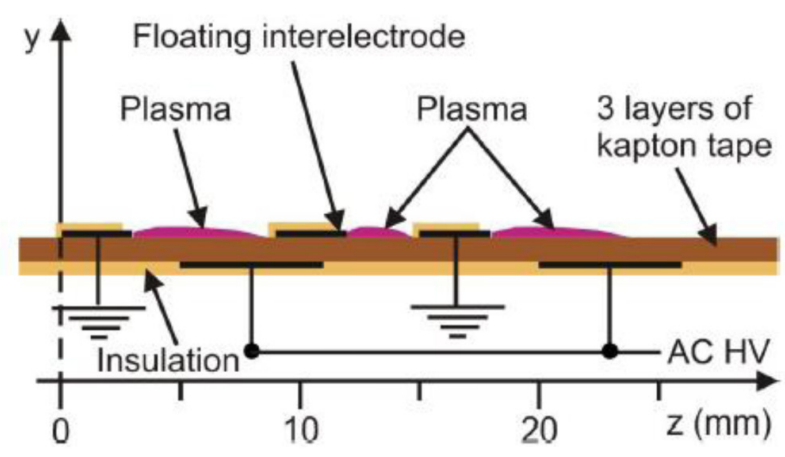

Fig. 4. Multi-electron system [12]

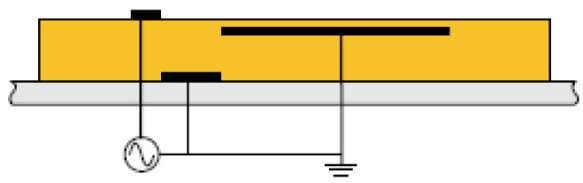

(a) Case 1

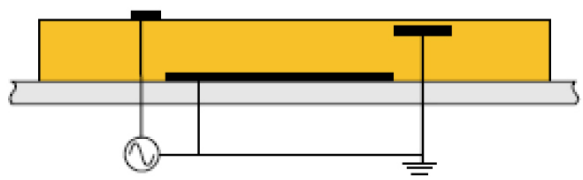

(b) Case 2
In addition, numerous modifications of multielectrode systems are introduced, most often they are electrodes in the form of copper strips that are placed at various depths in the dielectric layers; such plasma actuator construction is shown in Figure 5.

In the presented designs of plasma actuators, electrodes in the form of parallel strips were used most often. The research on the influence of electrode geometry on the operation of plasma actuators was conducted as well. The research conducted by Wang CC. [20], and Roy S. [21] show the influence of the shape of electrodes on the operation of the plasma actuator.

The shape of the plasma actuator electrodes directly influences the process of vortex production. During the operation of the DBD system with the linear shape of the electrodes, it produces much weaker vortex compared to a plasma actuator with spiral or square electrodes. The greater vorticity of systems with spiral and square electrodes results from the three-dimensional arrangement of the electrodes and hence the threedimensional nature of the discharges. Spiral geometry and square electrodes generate vorticity with three-dimensional mixing.

Additionally, the tests were carried out with a sawtooth electrode, the shape and dimensions of the electrode were shown in Figure 7 [18].

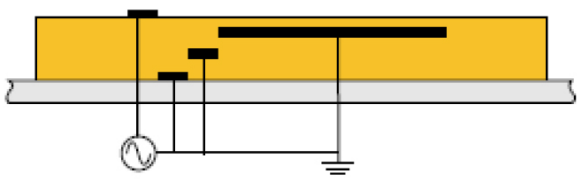

(c) Case 3

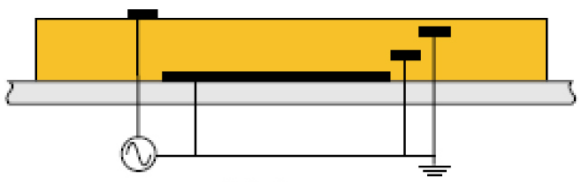

(d) Case 4

Fig. 5. Multi-electron system (grounded electrodes are located inside the dielectric) [19]

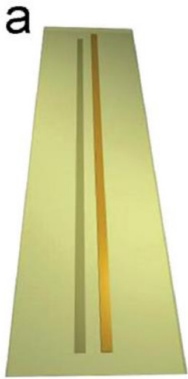

Linear

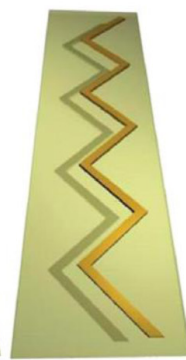

Triangular

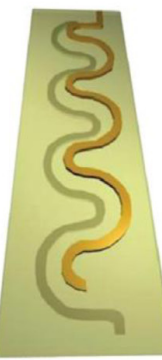

Serpentine
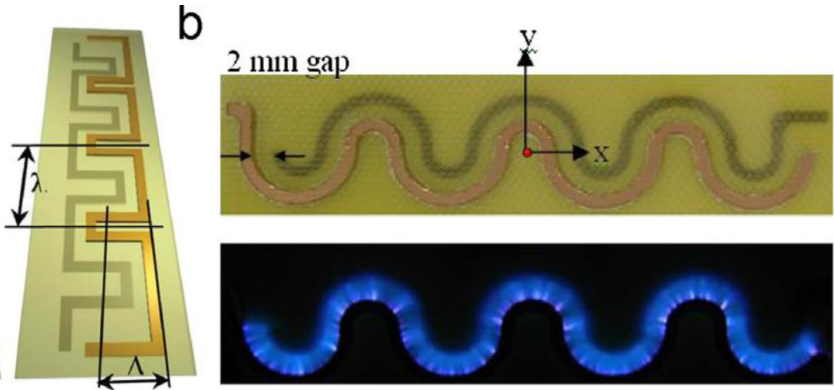

Square

Fig. 6. Schematics of different types of three-dimensional plasma actuators (a) [20], and the discharges for a serpentine actuator (b) [21] 


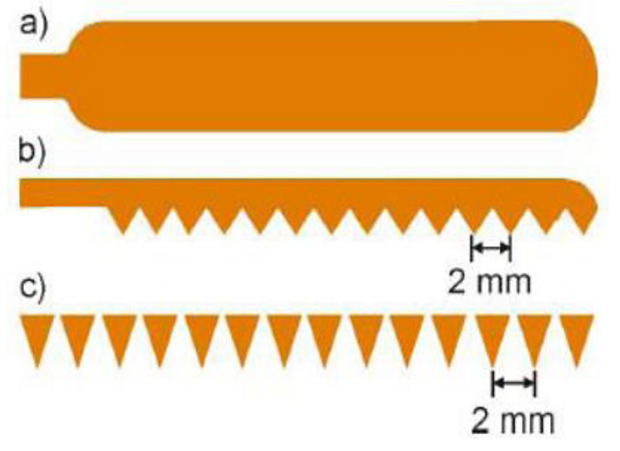

Fig. 7. Schematic top view of the smooth HV electrode (a), the saw-like grounded electrode (b) and separated saw teeth of the floating interelectrode (c) [18]

\section{EXPERIMENTAL RESEARCH OF PLASMA ACTUATOR WITH TWO MESH ELECTRODES ON THE WING MODEL}

The previous part of the manuscript presents the construction and operating principle of selected plasma actuators designs with an asymmetrical electrode system. This section presents the results of an experimental research involving the wing model with the dimensions of $250 \times 250 \mathrm{~mm}$ and SD 7003 profile. Experimental tests were carried out in the wind tunnel at a angle of attack of 15 degrees, air velocity of $U$ $=5-15 \mathrm{~m} / \mathrm{s}$ for the Reynolds number in range of 100000-262500. The DBD plasma actuator was placed directly on the surface of the wing model at a distance of $15 \mathrm{~mm}$ from the leading edge (high voltage electrode with dimensions of $10 \times 250 \mathrm{~mm}$ ). The experimental studies involved mesh electrodes with $0.05 \times 0.05 \mathrm{~mm}$ mesh sizes (grounded and high voltage electrodes) separated by a Kapton dielectric. The grounded electrode is located on $70 \%$ of the upper surface of the wing starting from the leading edge to the trailing edge. The difference in the design and principle of operation pertaining to a plasma actuator with an asymmetrical electrode system and a plasma actuator with two mesh electrodes is shown in Figure 8b.

The Keysight oscilloscope and equipment were used to measure the voltage and current of the DBD discharges. Registered oscillograms of the Lissajous figure allowed to calculate the power delivered to the DBD plasma actuator, selected Lissajous figures are shown in Figure 9 at $V=12.5 \mathrm{kV}$ and $V=13.5 \mathrm{kV}$ power supply voltage. In the experimental studies carried out, the discharge power was in the range of $P$ $=1.0 \mathrm{~W}$ to $2.2 \mathrm{~W}$, with the supply voltage from
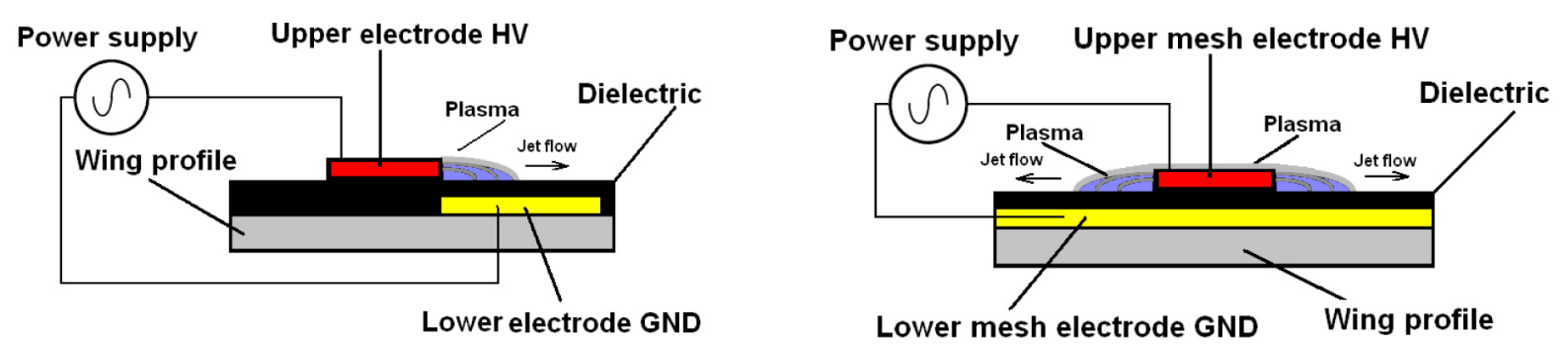

Fig. 8. Design and principle of DBD plasma actuator operation: a) with asymmetrical electrode system, b) with two mesh electrodes
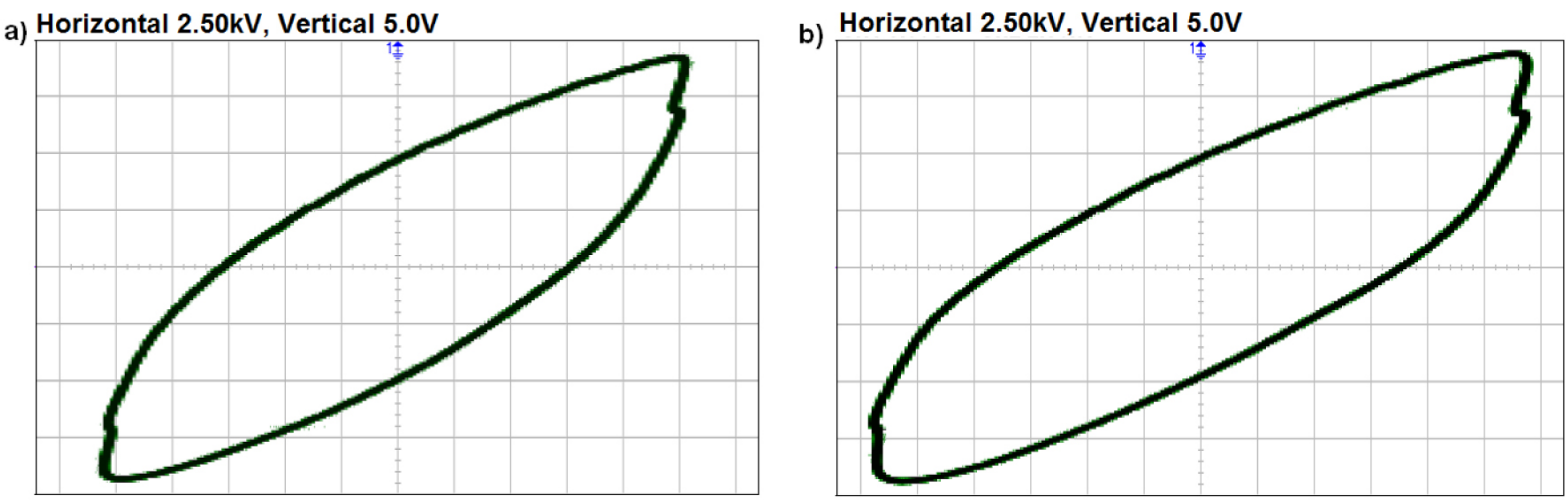

Fig. 9. Lissajous Figures recorded during the experimental research: a) for a $12.5 \mathrm{kV}$ supply voltage and a $7.5 \mathrm{kV}$ ignition voltage, b) $13.5 \mathrm{kV}$ supply voltage and a $8.8 \mathrm{kV}$ ignition voltage 
$V=7.0 \mathrm{kV}$ to $14 \mathrm{kV}$. The power of discharges in DBD systems is influenced by several factors; the most important ones include: power supply frequency, dielectric type [22], distance between electrodes, electrode geometry $[23,25]$. In the experimental tests carried out, a high-voltage power supply operating at a frequency of $f=$ $50 \mathrm{~Hz}$ was used to power the DBD plasma actuator. Figure 10 shows tunnel images of the wing model with SD 7003 profiles for the angle of attack $\alpha=15$ degrees and flow velocity $U=$ $5 \mathrm{~m} / \mathrm{s}$. Figure 10a shows turbulence in the form of vortices in a system with the plasma actuator switched off. Figure $10 \mathrm{~b}$ presents experimental research carried out with the plasma actuator turned on; there is no visible vortex formed after the separation point, flow is less turbulent.

The comparison of the working difference and discharge surface for a plasma actuator with asymmetrical electrode system and a plasma actuator with two mesh electrodes is shown in Figure 11. Using a plasma actuator with two mesh electrodes allows for discharges at the two edges of the electrode and through the high voltage electrode.

The comparison of the plasma actuator efficiency with two mesh electrodes placed on wing model was expressed by a percentage increase in aerodynamic force comparing the system operation with turned off and on plasma actuator under the same conditions, air flow velocity and the angle of attack, by measuring the acting forces. The percentage increase of aerodynamic force for the wing model with two mesh electrodes, for the angle of attack of $\alpha=15$ degrees and the air velocity in the range of $U=5-15 \mathrm{~m} / \mathrm{s}$, is shown in Figure 12.

The graph in Figure 12 shows the percentage increase in the lift force of the wing model with plasma actuator turned on. With increasing air flow velocity, the carrier force drops (profit expressed in percent decreases). The lift coefficient $C_{L}$ was used to determine the changes in lift force. Figure 13 shows the change in the lift coefficient of the wing model with plasma actuator turned off and on.

The analysis of the graph presented in Figure 12 shows a clear decrease in the percentage increase of the aerodynamic force (resultant force) along with the increase in the air velocity. For the air flow velocity in the range from $\mathrm{U}=5 \mathrm{~m} / \mathrm{s}$ to $10 \mathrm{~m} / \mathrm{s}$ and angle of attack of $\alpha=$ 15 degrees, the highest increase in the lift coefficient of $4 \%$ was obtained. With the increase of the air flow rate, the efficiency decreased. This effect should be explained by the lowered performance of the DBD system related to the low

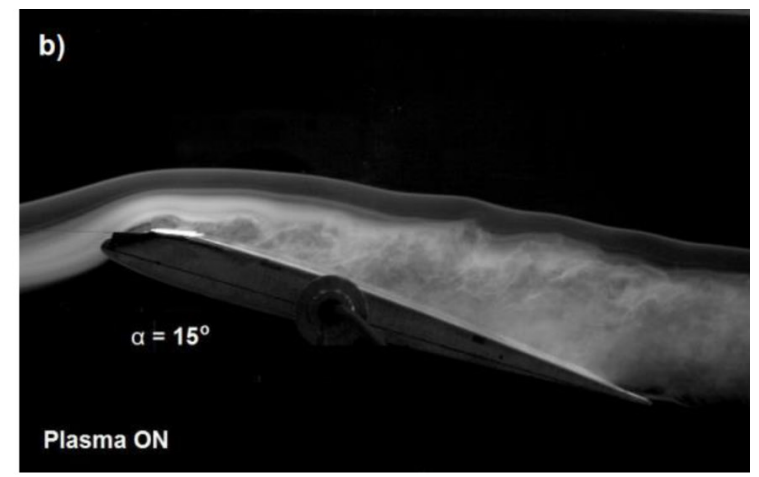

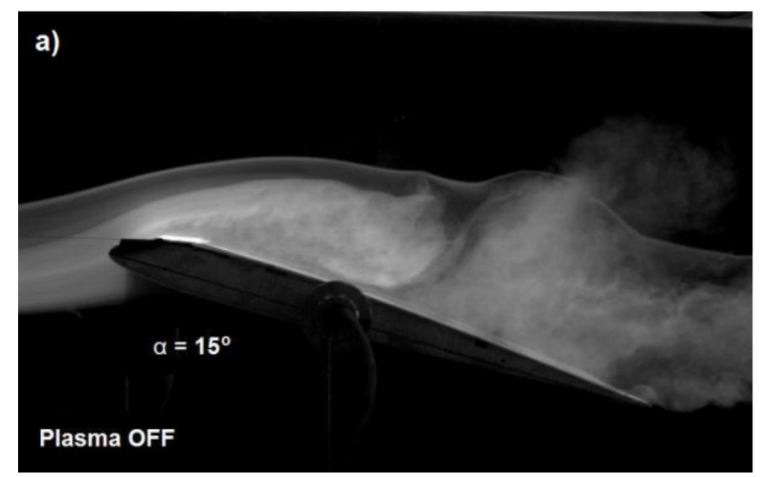

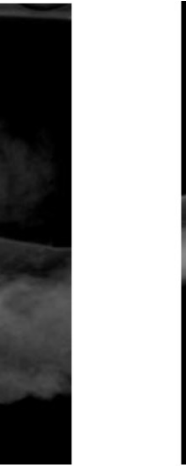

Fig. 10. Tunnel photos taken for SD7003 wing profile with velocity of $U=5 \mathrm{~m} / \mathrm{s}$; for attack angle $\alpha=15$ degrees: a) plasma actuator turned off, (visible turbulence), b) turned on plasma actuator, reduced turbulence

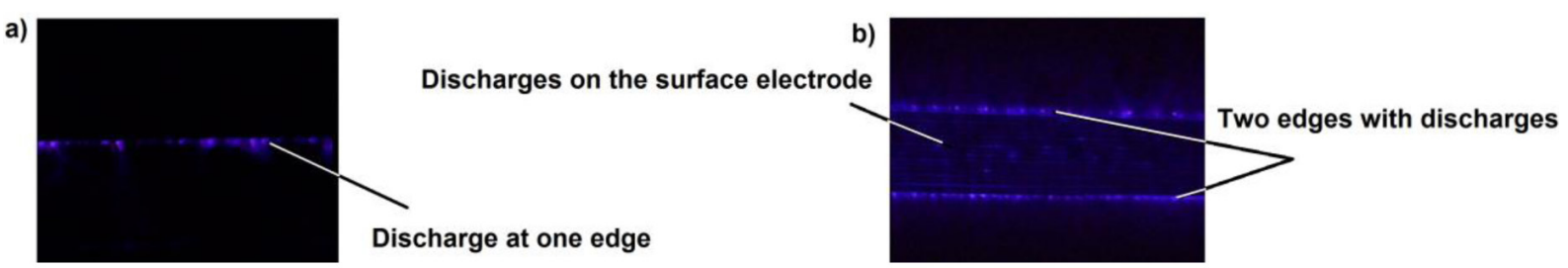

Fig. 11. Comparison of DBD plasma actuator discharges: a) asymmetric electrode system, with one visible edge of discharge, b) plasma actuator with two mesh electrodes, visible discharges at two edges and across high voltage electrodes 


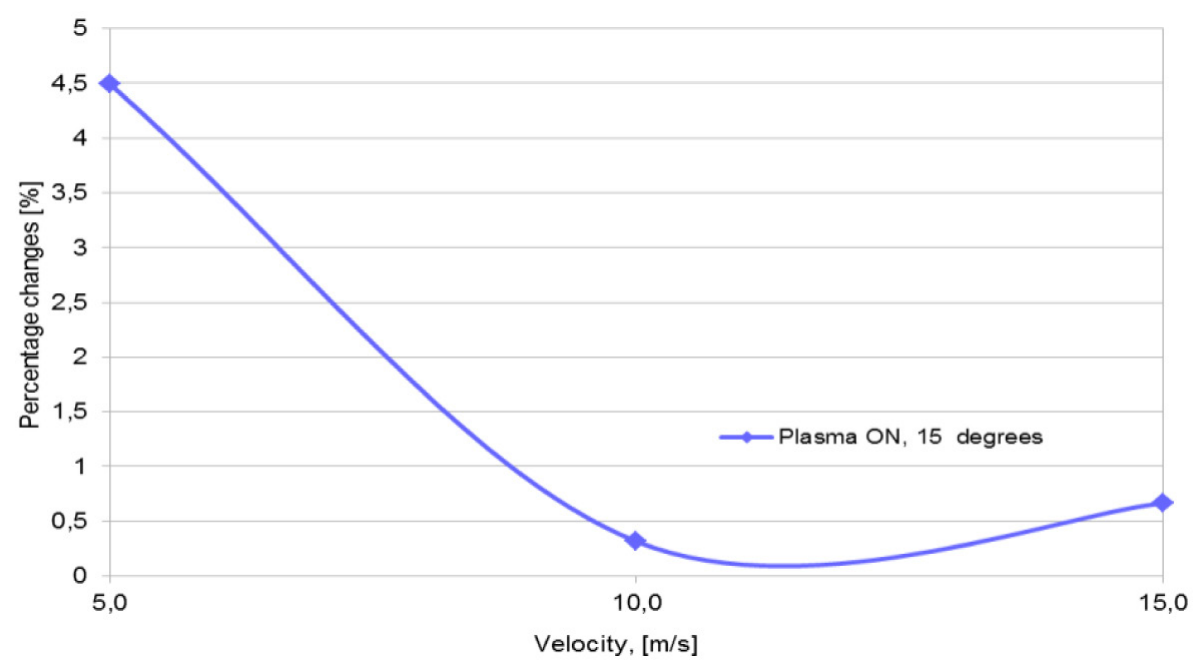

Fig. 12. Changes in the percentage increase in lift (profit), for the angle of attack $\alpha=15$ degrees depending on the air flow velocity

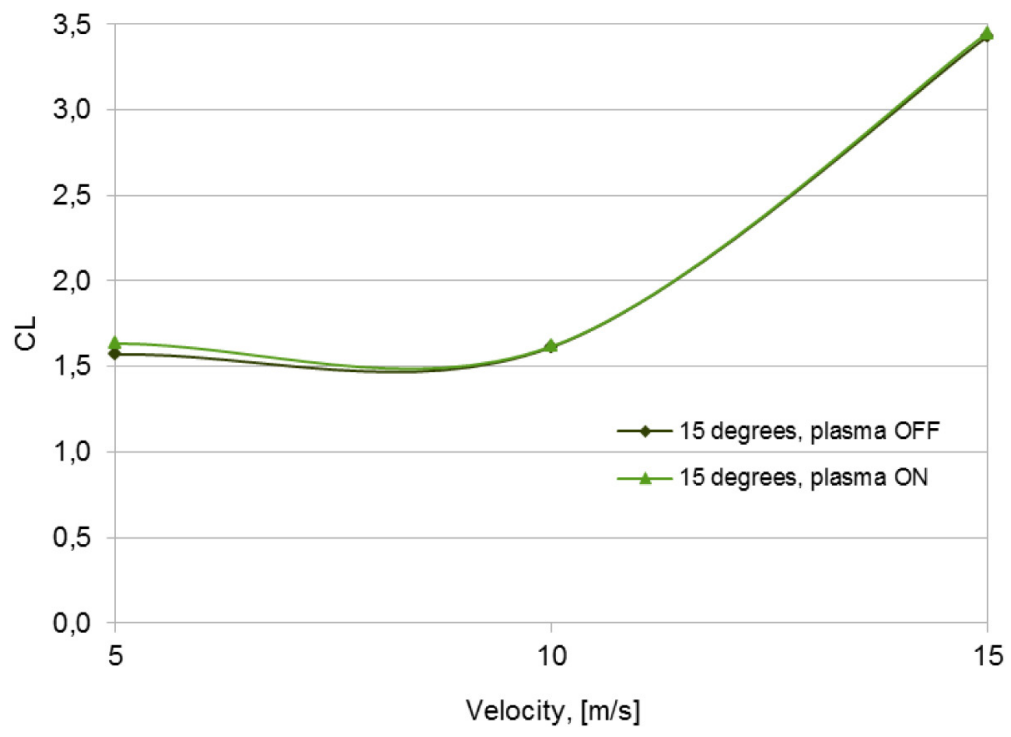

Fig. 13. Changes in the lift coefficient, for the angle of attack $\alpha=15$ degrees depending on the air flow velocity for the plasma actuator switched on and off

frequency of the power supply system and the increase in the air velocity. Similarly, in DBD ozonators, an increase in gas flow velocity at low feed frequencies results in a decrease in ozone concentration. In order to ensure high efficiency, it is necessary to increase the frequency of power supply systems.

\section{CONCLUSION}

The manuscript describes DBD plasma actuators the operation of which is based mainly on the production of the "ion wind". The power supply system significantly influences the operation of the plasma actuator, especially the frequency of the power supply. The operation of a DBD plasma actuator with two mesh electrodes is visible in tunnel photos showing improved flow laminarity in a system with a plasma actuator on. The effectiveness of operation depends also on the angle of attack of the airfoil as well as on the speed of air flow around the wing, as shown in Figure 13. This solution is a relatively new approach to influencing the air flow in the boundary layer; therefore, it requires a lot of research and optimization before it is implemented. 


\section{REFERENCES}

1. Wenzinger C.J. and Rogall F.M., Resume of AirLoads on Slats and Flaps; NACA Report No. 690, 1939, National Advisory Committee for Aeronautics: Washington, DC, USA.

2. Traub L.W. and Kaula Ma.P., Effect of LeadingEdge Slats at Low Reynolds Numbers, Aerospace Engineering Department, Embry Riddle Aeronautical University, Prescott, AZ 86301, USA.

3. Rudolph P.K.C., High-Lift Systems on Commercial Subsonic Airliners, 1996, NASA Contractor Report 4746.

4. Moraris V.M. and Lawson N.J., Garry K.P., Aerodynamic and Performance Characteristics of a Passive Leading Edge Kruger Flap at Low Reynolds Numbers. The Aeronautical Jurnal, 2012, 116(1181), 759-769.

5. Akaydin H.D., Housman J.A., Kiris C.C., Bahr Ch.J., Hutcheson F.V., Computational Design of a Krueger Flap Targeting Conventional Slat Aerodynamics, 2016, 22nd AIAA/CEAS Aeroacoustics Conference, Aeroacoustics Conferences.

6. Kumar M.S. and Kumar K.N., Design and Computational Studies on Plain Flaps, Bonfring. International Journal of Industrial Engineering and Management Science, 2013, 3(2), 33-39.

7. Solfelt D. and Maple R., CFD Analysis of a T-38 Wing Fence, 46th AIAA Aerospace Sciences Meeting and Exhibit, Aerospace Sciences Meetings, 7-10 January 2008, Reno, Nevada.

8. Meftah S.M.A., Belhenniche M., O Madani Fouatih, Imine B., Effects of Wing-Cuff on NACA 23015 Aerodynamic Performances, 2014, EPJ Web of Conferences 67, 02074.

9. Odilon V., Cavazos Jr, A Flow Visualization Study of Lex Generated Vortices on a Scale Model of a F/A-18 Fighter Aircraft at High Angles of Attack, Monterey, Californa 1990, AD-A236 534.

10. Pushpender S., Varma D., Ghosh S., Novel Vortex Generator for Mitigation of Shock-Induced Flow Separation, Journal of Propulsion and Power, 2011, 32(5), 1264-1274.

11. Gnapowski S., Gnapowski E., Duda A., Inproving of the quality food for animals by pulsed power plasma discharge, 2015, 9(27), 58-65, doi: $10.12913 / 22998624 / 59085$.

12. Gnapowski E., Gnapowski S., Pytka J., The impact of dielectrics on the electricalcapacity, concentration, efficiency ozonegeneration for the plasma reactor with meshelectrodes, Plasma Science and Technology, 2018, 20(8), 1-7, doi: 10.1088/2058$6272 /$ aac 1 b6.
13. Whalley R.D. and Choi K.S., The starting vortex in quiescent air induced by dielectric-barrier-discharge plasma. Journal of Fluid Mechanics, 2012, 703, 192-203.

14. Robinson M., A History of the Electric Wind. American Journal of Physics 1962, 30, 366-372.

15. Gnapowski E., Review of Selected Methods for Increasing the Aerodynamic Force of the Wing, Adv. Sci. Technol. Res. J., 2019, 13(1), 60-67, doi: $10.12913 / 22998624 / 103858$.

16. Gnapowski E., Gnapowski S., Pytka J., Effect of Mesh Geometry on Power, Efficiency and Homogeneity of Barrier Discharges in the Presence of Glass Dielectric. IEEE Transactions on Plasma Science, 2018, 46, 3493-3498.

17. Little J., Takashima K., Nishihara M., Adamovich I., Samimy M., High Lift Airfoil Leading Edge Separation Control with Nanosecond Pulse Driven DBD Plasma Actuators, 5th Flow Control Conference, Chicago, 2010, AIAA 2010-4256.

18. Leroy A., Audier P., Podlinski J., Berendt A., Hong D., Mizeraczyk J., Enhancement of lift and drag performances of NACA0012 airfoil by multi-DBD plasma actuator with additional floating interelectrodes. International Symposium on Electrohydrodynamics, Gdańsk, Poland 2012.

19. Erfani R., Erfani T., Utyuzhnikov S.V., Kontis K., Optimisation of multiple encapsulated electrode plasma actuator. Aerospace Science and Technology, 2012, 26, 120-127.

20. Wang C.C., Durscher R., Roy S., Three-dimensional effects of curved plasma actuators in quiescent airJournal of Applied Physics, 2011, 42, 109117, 083305.

21. Roy S., Wang C.C., Bulk flow modification with horseshoe and serpentine plasma actuators, Journal of Physics D: Applied Physics, 2009, 42(3), 032004.

22. Gnapowski E., Gnapowski S., Changes in the Power Discharge in a Plasma Reactor Using Porous Versus Solid Dielectric Barriers and Meshes Electrodes, IEEE Transactions on Plasma, 2016, 44(10), 2079-2083.

23. Gnapowski E., Effect of Mesh Electrodes Geometry on the Ozone Concentration in the Presence of Micanite Dielectric, Adv. Sci. Technol. Res. J., 2018, 12(4), \76-80, doi: 10.12913/22998624/100340.

24. Vey S., Low Aspect Ratio Wing Flow Control at Low Reynolds Numbers, Doctoral dissertation, Technical University of Berlin, 2014.

25. Belan M. and Messanelli F., Compared ionic wind measurements on multi-tip corona and DBD plasma actuators. Journal of Electrostatics, 2015, 76, 278-287. 